\title{
Countably metacompact spaces in the constructible universe
}

\author{
by
}

Paul J. S zept y cki (Toronto, Ont.)

\begin{abstract}
We present a construction from $\diamond^{*}$ of a first countable, regular, countably metacompact space with a closed discrete subspace that is not a $G_{\delta}$. In addition some nonperfect spaces with $\sigma$-disjoint bases are constructed.
\end{abstract}

1. Introduction. There is what may be called a metatheorem in the arena of covering and separation properties of topological spaces. A collection of theorems holds under $V=L$ iff their appropriate variants hold under PMEA. W. G. Fleissner and P. Nyikos were the first to establish this theme when in 1974 and 1980 they respectively proved:

$[\mathrm{F}](V=L) \Rightarrow$ Normal spaces of character $\leq 2^{\aleph_{0}}$ are collectionwise Hausdorff (henceforth abbreviated cwH).

[N1] $(\mathrm{PMEA}) \Rightarrow$ Normal spaces of character $<2^{\aleph_{0}}$ are collectionwise normal.

Soon after, S. Watson and D. K. Burke extended the analogy to the class of regular, countably paracompact spaces.

[W] $(V=L) \Rightarrow$ Countably paracompact regular spaces of character $<2^{\aleph_{0}}$ are $\mathrm{cwH}$.

[B] $(\mathrm{PMEA}) \Rightarrow$ Countably paracompact regular spaces of character $<2^{\aleph_{0}}$ are strongly cwH.

In the same paper Burke also proved the interesting result that under PMEA, in countably metacompact $T_{1}$ spaces of character $<2^{\aleph_{0}}$ closed discrete subspaces are $G_{\delta}$ 's. Burke raised the natural question whether the large cardinal inherent in the PMEA assumption was necessary. Recently Nyikos proved that under $V=L$ Burke's result holds in the class of lo-

1991 Mathematics Subject Classification: 03E45, 54A35, 54D15, 54G20.

Key words and phrases: countably metacompact, $G_{\delta}, \diamond^{*}$. 
cally countable spaces and asked if the full result holds in $L$ (see [N2]). We answer Nyikos's question in the negative by constructing a counterexample from $\diamond^{*}$.

F. D. Tall and Fleissner proved the Easton forcing analogues of the normal and countably paracompact results mentioned above (see [T1] and [T2]) and it is shown in [S] that Nyikos's result on locally countable, countably metacompact spaces similarly extends. However, Burke's original question remains unanswered, and in particular it is an interesting open problem to establish whether the Easton models answer it. For example, we were unable to answer

QUESTION 1.1. In the model obtained by adding $\omega_{2}$ many Cohen subsets of $\omega_{1}$ over a model of $C H$ (or $V=L$ ) in first countable, countably metacompact, regular (or even $T_{1}$ ) spaces of size $\omega_{1}$, are closed discrete sets $G_{\delta}$ 's?

Note that $\diamond^{*}$ does not hold in this model (see [K], VII, exercise J5). The role of the continuum in this problem is not well understood. For example it is not known whether the existence of an example implies the existence of one of size $\mathfrak{c}$. Burke's PMEA proof may be interpreted, as in [DTW], as a forcing and reflection proof. If you add Random or Cohen reals over a model of $\mathrm{CH}$ there will be no counterexamples of size less than the continuum. Adding supercompact many reals assures that there are no examples of size greater than or equal to the continuum. The supercompact cardinal allows one to prove that if there were one of size greater than or equal to $\mathfrak{c}$, then there would also be an example of size less than $\mathbf{c}$. However, there may be a weaker ZFC reflection result which would, along with a positive answer to 1.1, answer Burke's question.

QUESTION 1.2. If there is a regular, countably metacompact space with a closed discrete set which is not a $G_{\delta}$, then is there also one of size $\mathfrak{c}$ ?

In Section 4 we present a related construction of some spaces with $\sigma$ disjoint bases and closed discrete sets that are not $G_{\delta}$ 's. We would like to thank Steve Watson for pointing out the method of splitting points to construct these examples which inspired many simplifications and the final form of the main example of Sections 2 and 3.

2. Preliminaries. Our notation and terminology are fairly standard. We refer the reader to $[\mathrm{K}]$ for any unfamiliar notions.

Let $\left\{g_{\alpha}: \alpha<\omega_{1}\right\}$ be a $\diamond$ sequence on $\omega_{1} \times \omega_{\omega}$. That is, for every $g$ : $\omega_{1} \times \omega \rightarrow \omega,\left\{\alpha: g\left\lceil\alpha \times \omega=g_{\alpha}\right\}\right.$ is stationary. In addition, let $\left\{\mathcal{B}_{\alpha}: \alpha<\omega_{1}\right\}$ be a $\diamond^{*}$ sequence in the following sense. For each $\alpha<\omega_{1}, \mathcal{B}_{\alpha}$ is a countable collection from ${ }^{\alpha} \omega$ such that for every countable partition $H: \omega_{1} \rightarrow \omega$ of $\omega_{1},\left\{\alpha: H\left\lceil\alpha \in \mathcal{B}_{\alpha}\right\}\right.$ contains a club. 
For a subset $\mathcal{F} \subseteq \operatorname{Fn}\left(\omega_{1}, \omega, \omega_{1}\right)$ we define a topology on $X_{\mathcal{F}}=\omega_{1} \cup \mathcal{F}$. Declare each point of $\mathcal{F}$ isolated and for each $\alpha \in \omega_{1}$ define

$$
U_{n}(\alpha)=\{\alpha\} \cup\{f \in \mathcal{F}: \alpha \in \operatorname{dom}(f), f(\alpha) \geq n\} .
$$

And for each $\alpha$ we let $\left\{U_{n}(\alpha): n<\omega\right\}$ be a local neighborhood base at $\alpha$. We will choose $\mathcal{F}$ with some care to ensure that the resulting $X_{\mathcal{F}}$ will be zero-dimensional and countably metacompact and $\omega_{1}$ will not be a $G_{\delta}$.

The following is a standard formulation of countable metacompactness.

LEMMA. $X_{\mathcal{F}}$ is countably metacompact if and only if for every partition $H: \omega_{1} \rightarrow \omega$ of $\omega_{1}$, there is a point finite family $\left\{W_{n}: n<\omega\right\}$ of open sets such that for each $n<\omega, W_{n} \supseteq H^{-1}(n)$.

Notice that by definition $X_{\mathcal{F}}$ is first countable and $T_{1}$; moreover, $\mathcal{F}$ consists of isolated points and $\omega_{1}$ is closed discrete.

To assure later that the space will be zero-dimensional we fix two functions $\Delta, e: \omega_{1}^{2} \rightarrow \omega$. For each $\alpha, \Delta$ will tell us for $\beta<\alpha$ which neighborhood of $\beta$ is disjoint from $U_{1}(\alpha)$ while $e$ will tell us the same for those $\beta>\alpha$. This will be encapsulated in clauses (d) and (e) of Definition 3.1.

For each limit ordinal $\alpha<\omega_{1}$, fix an increasing sequence $\left\{\xi_{n}: n<\omega\right\}$ cofinal with $\alpha$ and for each $\beta<\alpha$ let

$$
e(\beta, \alpha)=n \quad \text { iff } \quad \beta \in\left[\xi_{n}, \xi_{n+1}\right) .
$$

For each successor ordinal $\alpha$ and each $\beta<\alpha$, let

$$
e(\beta, \alpha)=0 \text {. }
$$

For $\beta \geq \alpha, e(\beta, \alpha)$ is neither defined nor used.

For the definition of $\Delta$ we need to fix an enumeration $A=\left\{a_{\alpha}: \alpha<\omega_{1}\right\}$ of any uncountable subset of $\omega_{\omega}$ and let

$$
\Delta(\beta, \alpha)=\min \left\{n<\omega: a_{\alpha}(n) \neq a_{\beta}(n)\right\} .
$$

\section{The construction}

Definition 3.1. For each $g_{\alpha}$ from the $\diamond$ sequence and $\mathcal{B}_{\alpha}$ from the $\diamond^{*}$ sequence we say that $f$ diagonalizes $\left(g_{\alpha}, \mathcal{B}_{\alpha}\right)$ if

(a) $\operatorname{dom}(f)=\left\{\alpha_{n}: n<\omega\right\}$ is an increasing sequence of limit ordinals and $\sup \left\{\alpha_{n}: n<\omega\right\}=\alpha$.

(b) For each $n<\omega, f\left(\alpha_{n}\right)=g_{\alpha}\left(\alpha_{n}, n\right)$.

(c) If $H \in \mathcal{B}_{\alpha}$ then either $H$ eventually dominates $f$, or is eventually constant on the domain of $f$, i.e., either $\exists N<\omega$ such that $\forall n>N, f\left(\alpha_{n}\right)<$ $H\left(\alpha_{n}\right)$, or $\exists M<\omega, \operatorname{dom}(f) \subseteq{ }^{*} H^{-1}(M)$.

(d) $\forall n<m, f\left(\alpha_{n}\right)<\Delta\left(\alpha_{n}, \alpha_{m}\right)$.

(e) $\forall n<m, f\left(\alpha_{m}\right)<e\left(\alpha_{n}, \alpha_{m}\right)$. 
Let $G=\left\{\alpha: \exists f, f\right.$ diagonalizes $\left.\left(g_{\alpha}, \mathcal{B}_{\alpha}\right)\right\}$. For each $\alpha \in G$ fix $f_{\alpha}$ witnessing it and let $\mathcal{F}=\left\{f_{\alpha}: \alpha \in G\right\}$.

First note that by construction every successor ordinal in $\omega_{1}$ is isolated in $X_{\mathcal{F}}$. This follows from the fact that the domain of any diagonalizing function consists entirely of limit ordinals.

LemMa 3.2. $X_{\mathcal{F}}$ is zero-dimensional. Furthermore, it is pseudonormal, i.e., any two disjoint closed sets, one of which is countable, can be separated by open sets.

Proof. To prove that $X_{\mathcal{F}}$ is zero-dimensional, it suffices to show that for any $\alpha<\omega_{1}$ and $i \geq 1, U_{i}(\alpha)$ is clopen.

Claim 1. For $\beta<\alpha$ and $i \geq 1, U_{i}(\alpha) \cap U_{\Delta(\beta, \alpha)}(\beta)=\emptyset$.

Suppose not and fix $\gamma \in G$ and suppose $f_{\gamma} \in U_{i}(\alpha) \cap U_{\Delta(\beta, \alpha)}(\beta)$. Let $\left\{\gamma_{m}: m<\omega\right\}$ be the increasing enumeration of $\operatorname{dom}\left(f_{\gamma}\right)$. There must be integers $n<m$ such that $\beta=\gamma_{n}$ and $\alpha=\gamma_{m}$. Since $f_{\gamma}$ is a diagonalizing function, $f_{\gamma}\left(\gamma_{n}\right)<\Delta\left(\gamma_{n}, \gamma_{m}\right)$ must hold by (d). But this implies that $f_{\gamma} \notin U_{\Delta(\beta, \alpha)}(\beta)$. Contradiction.

Claim 2. For $\beta>\alpha$ and $i \geq 1, U_{e(\alpha, \beta)}(\beta) \cap U_{i}(\alpha)=\emptyset$.

As in Claim 1, suppose for some $\gamma \in G, f_{\gamma} \in U_{e(\alpha, \beta)}(\beta) \cap U_{i}(\alpha)$. Fix $n<m$ such that $\gamma_{n}=\beta$ and $\gamma_{m}=\eta$. Then by (e), we have $f_{\gamma}\left(\gamma_{m}\right)<$ $e\left(\gamma_{n}, \gamma_{m}\right)=e(\alpha, \beta)$, which implies that $f_{\gamma} \notin U_{e(\alpha, \beta)}(\beta)$. Contradiction.

Finally, to see that $X_{\mathcal{F}}$ is pseudonormal, it suffices to show that for every $\alpha<\omega_{1}$, there is a clopen $U$ such that $U \cap \omega_{1}=\alpha$. Fix $\alpha<\omega_{1}$ and let

$$
U=\bigcup_{\beta<\alpha} U_{\Delta(\beta, \alpha)}(\beta) \text {. }
$$

Clearly, by Claim $1, U_{1}(\alpha) \cap U=\emptyset$. For each $\beta<\alpha$ and each $\eta>\alpha$,

$$
e(\beta, \eta) \leq e(\alpha, \eta) \text {. }
$$

Therefore, along with Claim 2, this implies that for each $\eta>\alpha, U \cap$ $U_{e(\alpha, \eta)}(\eta)=\emptyset$. Therefore $U$ is clopen and clearly $U \cap \omega_{1}=\alpha$.

LEMMA 3.3. $X_{\mathcal{F}}$ is countably metacompact.

Proof. Fix $H: \omega_{1} \rightarrow \omega$. It suffices to find open sets $W(n) \supseteq H^{-1}(n)$ such that $\{W(n): n<\omega\}$ is point finite.

Fix a club $C$ such that $\forall \alpha \in C, H\left\lceil\alpha \in \mathcal{B}_{\alpha}\right.$. For each $\alpha \in C$ let $\alpha(+)$ be the successor of $\alpha$ in $C$. For each $\alpha \in C,[\alpha, \alpha(+))$ is a countable closed discrete subset of $X_{\mathcal{F}}$, so we can separate it by open sets. Therefore fix $F_{\alpha}:[\alpha, \alpha(+)) \rightarrow \omega$ such that both

(i) for each $\beta \neq \gamma$ in $[\alpha, \alpha(+)), U_{F_{\alpha}(\beta)}(\beta) \cap U_{F_{\alpha}(\gamma)}=\emptyset$, and

(ii) for each $\beta \in[\alpha, \alpha(+)), F_{\alpha}(\beta)>H(\beta)$. 
Let $F=\bigcup_{\alpha \in C} F_{\alpha}$. Then $F$ codes a neighborhood assignment by

$$
W(n)=\bigcup\left\{U_{F(\beta)}(\beta): H(\beta)=n\right\} .
$$

Clearly, for each $n, W(n) \supseteq H^{-1}(n)$ so it suffices to verify that $\{W(n)$ : $n<\omega\}$ is point finite. If not, there is a $f_{\gamma} \in \mathcal{F}$ such that

$$
B=\left\{n<\omega: f_{\gamma} \in W(n)\right\}
$$

is infinite. For each $n \in B$ there is a $\gamma(n)$ such that $f_{\gamma} \in U_{F(\gamma(n))}(\gamma(n))$ and $H(\gamma(n))=n$. This implies that for no $M<\omega$ is it the case that $\operatorname{dom}\left(f_{\gamma}\right) \subseteq^{*} H^{-1}(M)$, and

(iii) $\forall n \in B, f_{\gamma}(\gamma(n))>F(\gamma(n))$.

Furthermore, (i) implies that

(iv) $\forall \alpha \in C,|[\alpha, \alpha(+)) \cap\{\gamma(n): n \in B\}| \leq 1$.

Now, (iv) implies that $\sup \{\gamma(n): n \in B\} \in C$, hence that $\gamma \in C$. Therefore, since $f_{\gamma}$ diagonalizes $\left(g_{\gamma}, \mathcal{B}_{\gamma}\right)$ and since $H\left\lceil\gamma \in \mathcal{B}_{\gamma}\right.$, (c) implies that for all but finitely many $n \in B, f_{\gamma}(\gamma(n))<H(\gamma(n))$. This contradicts (ii) and (iii).

LEMMA 3.4. $\omega_{1}$ is not a $G_{\delta}$ in $X_{\mathcal{F}}$.

Proof. If $\{W(n): n<\omega\}$ is a sequence of open neighborhoods of $\omega_{1}$, then for each $\alpha \in \omega_{1}$ and $n<\omega$, fix $g(\alpha, n)$ minimal so that $U_{g(\alpha, n)}(\alpha) \subseteq$ $W(n)$. This defines $g: \omega_{1} \times \omega \rightarrow \omega$. Suppose we could find an $\alpha \in G$ such that if $\left\{\alpha_{n}: n<\omega\right\}$ is the increasing enumeration of $\operatorname{dom}\left(f_{\alpha}\right)$ then for each $n<\omega, f_{\alpha}\left(\alpha_{n}\right)=g\left(\alpha_{n}, n\right)$. This would imply that for each $n<\omega$, $f_{\alpha} \in U_{g\left(\alpha_{n}, n\right)}\left(\alpha_{n}\right)$ and in particular $f_{\alpha} \in \bigcap_{n<\omega} W(n)$. Therefore it suffices to prove

LEMma 3.5. There is an $\alpha \in G$ such that $g\left\lceil\alpha \times \omega=g_{\alpha}\right.$.

Proof of 3.5. Let $(*)_{\alpha}$ be the following statement:

$(*)_{\alpha} \quad$ There is an increasing sequence $\left\{\beta_{n}: n<\omega\right\}$ of limit ordinals cofinal in $\alpha$ such that

(i) $\forall i \leq n, g_{\alpha}\left(\beta_{n}, i\right)=g_{\alpha}\left(\beta_{i}, i\right)$.

(ii) $\forall j \leq i<n, g_{\alpha}\left(\beta_{j}, j\right)<\Delta\left(\beta_{i}, \beta_{n}\right)$.

(iii) $\forall j \leq n \forall i<n, g_{\alpha}\left(\beta_{j}, j\right)<e\left(\beta_{i}, \beta_{n}\right)$.

The proof of 3.5 splits up into two steps.

LEMMA 3.6. For any $g: \omega_{1} \times \omega \rightarrow \omega,\left\{\alpha: g\left\lceil\alpha=g_{\alpha}\right.\right.$ and $(*)_{\alpha}$ holds $\}$ is stationary.

LEMMA 3.7. If $(*)_{\alpha}$ holds then $\alpha \in G$.

Proof of 3.6. We will construct sequences of

- stationary sets $Y_{0} \supseteq Y_{1} \supseteq \ldots \supseteq Y_{i} \supseteq \ldots$, 
- ordinals $\gamma_{0}<\gamma_{1}<\ldots<\gamma_{i}<\ldots$, and

- integers $\left\{n_{i}: i<\omega\right\}$

so that, letting $N_{i}=\max \left\{n_{j}: j \leq i\right\}$ :

(1) $\forall i \forall \alpha \in Y_{i}, g(\alpha, i)=n_{i}$.

(2) $\forall i$, there is an $s \in{ }^{N_{i}} \omega$ such that for all $\alpha \in Y_{i}, s \subseteq a_{\alpha}$.

(3) $\forall i$, for every limit ordinal $\alpha \in Y_{i}, e_{\alpha}^{-1}\left(\left\{0, \ldots, n_{i}\right\}\right) \subseteq \gamma_{i}$.

Note that clause (2) says that $\Delta(\alpha, \beta)>N_{i}$ for every $\alpha, \beta \in Y_{i}$.

Pick $n_{0}$ so that $g^{-1}\left(n_{0}\right) \cap \omega_{1} \times\{0\}=X_{0} \times\{0\}$ is stationary. Fix $\gamma_{0}$ by the pressing down lemma so that $Z_{0}=\left\{\alpha \in X_{0}: \alpha\right.$ is a limit and $e_{\alpha}^{-1}(\{0, \ldots$ $\left.\left.\left.\ldots, n_{0}\right\}\right) \subseteq \gamma_{0}\right\}$ is stationary. Pick an $s \in{ }^{n_{0}+1} \omega$ so that $Y_{0}=\left\{\alpha \in Z_{0}: s \subseteq\right.$ $\left.a_{\alpha}\right\}$ is stationary. Having defined $Y_{k-1}, n_{k-1}$, and $\gamma_{k-1}$, define $Y_{k}$ similarly. Pick $n_{k}$ so that $g^{-1}\left(n_{k}\right) \cap Y_{k-1} \times\{k\}=X_{k} \times\{k\}$ is stationary. Fix $\gamma_{k}>\gamma_{k-1}$ by the pressing down lemma so that $Z_{k}=\left\{\alpha \in X_{k}: e_{\alpha}^{-1}\left(\left\{0, \ldots, n_{k}\right\}\right) \subseteq \gamma_{k}\right\}$ is stationary. Pick an $s \in{ }^{N_{k}} \omega$ so that $Y_{k}=\left\{\alpha \in Z_{k}: s \subseteq a_{\alpha}\right\}$ is stationary. Clearly by the construction, (1)-(3) are satisfied.

If $\gamma=\sup \left\{\gamma_{n}: n<\omega\right\}$ and $\beta_{0}<\beta_{1}<\ldots$ is any sequence of limit ordinals such that $\beta_{0}>\gamma$ and for each $i, \beta_{i} \in Y_{i}$, then by (1) we have

$$
\forall i<k<\omega, \quad g\left(\beta_{k}, i\right)=g\left(\beta_{i}, i\right)=n_{i} .
$$

By (3) and the fact that for each $j<k, e_{\beta_{k}}^{-1}\left(\left\{0, \ldots, n_{j}\right\}\right) \subseteq \gamma_{j}<\beta_{i}$, we have

$$
\forall j<k \forall i<k, \quad e\left(\beta_{i}, \beta_{k}\right)>N_{k}>g\left(\beta_{j}, j\right),
$$

and finally by (2), since for $i<k$ both $\beta_{i}$ and $\beta_{k}$ are in $Y_{i}$,

$$
\forall j \leq i<k, \quad \Delta\left(\beta_{i}, \beta_{k}\right)>N_{i} \geq g\left(\beta_{j}, j\right) .
$$

Now if we let $C_{i}=\left\{\alpha\right.$ : the limit ordinals in $Y_{i}$ are unbounded in $\left.\alpha\right\}$ and let $C=\bigcap_{i<\omega} C_{i}$, then $C$ is a club and for any $\alpha \in C$ we may find a sequence of limit ordinals $\beta_{i} \in Y_{i}$ cofinal in $\alpha$. Therefore since $\left\{g_{\alpha}: \alpha<\omega_{1}\right\}$ is a $\diamond$ sequence, the set of $\alpha$ from $C$ such that $f\left\lceil\alpha=g_{\alpha}\right.$ is stationary.

Proof of 3.7. Assume that $\alpha<\omega_{1}$ is such that $(*)_{\alpha}$ holds and fix $\left\{\beta_{m}\right.$ : $m<\omega\}$ witnessing it. Enumerate $\mathcal{B}_{\alpha}$ as $\left\{H_{n}: n<\omega\right\}$. Now we will choose a subsequence $\left\{\alpha_{m}: m<\omega\right\}$ of $\left\{\beta_{m}: m<\omega\right\}$ so that if $f$ is defined by

$$
f\left(\alpha_{m}\right)=g_{\alpha}\left(\alpha_{m}, m\right)
$$

then $f$ diagonalizes $\left(g_{\alpha}, \mathcal{B}_{\alpha}\right)$. To do this it suffices to find a subsequence $\left\{\alpha_{m}: m<\omega\right\} \subseteq\left\{\beta_{m}: m<\omega\right\}$ such that $\forall n<\omega$ either

(iv) $\exists M,\left\{\alpha_{m}: m<\omega\right\} \subseteq^{*} H_{n}^{-1}(M)$, or

(v) $\exists N_{n} \forall m>N_{n}, H_{n}\left(\alpha_{m}\right)>g\left(\alpha_{m}, m\right)$.

Note that (iv) and (v) imply clause (c) of the definition of a diagonalizing function; (ii) and (iii) of $(*)_{\alpha}$ imply that any subsequence of $\left\{\beta_{m}: m<\omega\right\}$ 
will satisfy clauses (d) and (e) respectively. The rest of the proof is devoted to the construction of the subsequence.

Notice that if $\left\{\eta_{m}: m<\omega\right\}$ is any subsequence of $\left\{\beta_{m}: m<\omega\right\}$ listed in increasing order then, by assumption on $\left\{\beta_{m}: m<\omega\right\}$, we have $g\left(\beta_{m}, m\right)=g\left(\eta_{m}, m\right)$.

First, by induction on $n$, define a sequence of infinite subsets of $\left\{\beta_{m}\right.$ : $m<\omega\}$ :

$$
X_{0} \supseteq X_{1} \supseteq \ldots \supseteq X_{n}
$$

so that for each $n$ either

$$
\exists i, X_{n} \subseteq H_{n}^{-1}(i), \quad \text { or } \quad \forall i, X_{n} \cap H_{n}^{-1}(i) \text { is finite. }
$$

Choose $X$ so that $\forall n, X \subseteq^{*} X_{n}$, and let $Z=\left\{k_{j}: j<\omega\right\}=\{n$ : $\forall i, X \cap H_{n}^{-1}(i)$ is finite $\}$. Then for each $n \notin Z$ there is an $i$ such that $X \subseteq{ }^{*} H_{n}^{-1}(i)$. By induction choose $\alpha_{n} \in X$ so that $\alpha_{0}<\ldots<\alpha_{n}<\ldots$ as follows. Choose $\alpha_{0} \in X$ so that $H_{k_{0}}\left(\alpha_{0}\right)>g\left(\beta_{0}, 0\right)$. Having $\alpha_{n-1}$ choose $\alpha_{n} \in X$ above $\alpha_{n-1}$ so that $\forall j \leq n, H_{k_{j}}\left(\alpha_{n}\right)>g\left(\beta_{n}, n\right)$ : as $X \cap H_{k_{j}}^{-1}(i)$ is finite for each $i$, this is easily done. It is easy for the reader to check that $(\mathrm{v})$ holds for those $n \in Z$ and (iv) holds otherwise. This completes the proof.

4. First countable spaces with $\sigma$-disjoint bases. In [D] Peter Davies constructed two first countable nonperfect spaces settling a question of Fleissner and Reed. One, constructed in ZFC, had a point countable base and a closed discrete set which witnessed that the space was nonperfect, while the other, constructed under $2^{\aleph_{0}}<2^{\aleph_{1}}$, had a $\sigma$-disjoint base but no uncountable closed discrete subsets. Fleissner and Reed [FR] proved that under MA every first countable space of cardinality less than the continuum possessing a $\sigma$-point finite base is perfect. These results leave open the status of closed discrete sets in spaces with $\sigma$-disjoint bases.

In this section we construct in ZFC a regular first countable space of cardinality $\mathfrak{c}$ with a $\sigma$-disjoint base containing a closed discrete set that is not a $G_{\delta}$. Furthermore, we prove it consistent that there is such an example of cardinality less than $\mathfrak{c}\left(^{1}\right)$.

Let $X=D \cup I$ be a first countable regular space of scattered height 2, where $I$ is the set of isolated points, and $D$ is closed discrete; for example, $\Psi(A)$ where $A$ is a maximal almost disjoint family of countable subsets of $\omega_{1}$ (see $[\mathrm{vD}]$ ). For another example see $[\mathrm{D}]$. For each $d \in D$ let $\left\{U_{n}(d): n<\omega\right\}$ be a neighborhood base at $d$. We let $\mathcal{F} \subseteq{ }^{\omega} D$ and "split" the points of $X$ to define a topology on $X_{\mathcal{F}}=(D \times \omega) \cup(I \times \mathcal{F})$ as follows.

$\left.{ }^{1}\right)$ J. Chaber has informed us that the ZFC example is very similar to Example 2.4 in $[\mathrm{C}]$; however, the example of size less than $\mathfrak{c}$ is new. 
Declare the points of $I \times \mathcal{F}$ isolated and for each $(d, n) \in D \times \omega$ and $m<\omega$ let

$$
U_{m}(d, n)=\{(d, n)\} \cup\left\{(i, f): i \in U_{m}(d), f \in \mathcal{F}, f(n)=d\right\} .
$$

$\mathrm{Claim}$. For any $X$ and $\mathcal{F}$ as above, $X_{\mathcal{F}}$ is first countable, zero-dimensional and has a $\sigma$-disjoint base. Furthermore, if $D$ is not a $G_{\delta}$ in $X$ then $D \times \omega$ is not $a G_{\delta}$ in $X_{\mathcal{F}}$.

Pro of. That $X_{\mathcal{F}}$ is zero-dimensional follows easily from the assumption that $X$ is, and $X_{\mathcal{F}}$ is first countable by definition. Notice that for each $n<\omega$ and each $e \neq d$ from $D, U_{1}(d, n) \cap U_{1}(e, n)=\emptyset$. So, letting $B_{n, m}=$ $\left\{U_{m}(d, m): d \in D\right\}$

$$
B=\bigcup_{n, m<\omega} B_{n, m} \cup(I \times \mathcal{F})
$$

is clearly a $\sigma$-disjoint base for $X_{\mathcal{F}}$.

Suppose that $D \times \omega$ is a $G_{\delta}$ and fix open sets $W_{n} \supseteq D \times \omega$ such that $\bigcap_{n<\omega} W_{n}=D \times \omega$. Then we may define sets $V_{n} \supseteq D$ open in $X$ by

$V_{n}=\bigcup\left\{U_{m_{d}}(d): m_{d}\right.$ is the minimal integer $k$ satisfying $\left.U_{k}(d, n) \subseteq W_{n}\right\}$.

Assuming $D$ is not a $G_{\delta}$ we may find an $i \in I \cap \bigcap_{n<\omega} V_{n}$. Therefore there is a sequence $\left\{d_{n}: n<\omega\right\}$ from $D$ such that for each $n<\omega, i \in U_{m_{d_{n}}}\left(d_{n}\right)$. Let $f \in{ }^{\omega} D$ be the function defined by $f(n)=d_{n}$. Then $(i, f) \in \bigcap_{n<\omega} W_{n}$. Contradiction.

To find an example of size less than the continuum we just take our example and add more than $\mathfrak{c}$ many Cohen or random reals. That our space in the extension has all the requisite properties follows from the next lemma. In the following, we say a topological property $\Phi$ is preserved by a notion of forcing $P$ if for every topological space $X$, if $\Phi(X)$ then $\Vdash_{P} \Phi(X)$.

Lemma 4.1. The following topological properties are preserved by Random and Cohen forcing:

(1) $X$ is first countable.

(2) $X$ is zero-dimensional.

(3) $X$ has a $\sigma$-disjoint base.

(4) $X$ is not perfect.

Proof. The proofs of (1)-(3) are trivial. For (4) we need to use the notion of endowments. For sake of brevity we present the Cohen real proof leaving the proof for Random reals to the interested reader. The next lemma, not stated in full generality, is due to Alan Dow. For a proof of it see [DTW].

Lemma 4.2. Let $S$ be a set and $n$ be a positive integer. For each $p \in$ $\operatorname{Fn}(S, 2)$, there exists a family $\mathcal{L}_{n}$ of finite subsets of $\operatorname{Fn}(S, 2)$ such that: 
(i) for each maximal antichain $A \subseteq \operatorname{Fn}(S, 2)$ below $p$ there is $L \in \mathcal{L}_{n}$ such that $L \subseteq A$,

(ii) for any element $f \in \operatorname{Fn}(S, 2)$ below $p$ with domain of size $n$ and any element $L \in \mathcal{L}_{n}$ there exists $g \in L$ such that $f$ and $g$ are compatible.

We call the family $\mathcal{L}_{n}$ an $n$-dowment below $p$.

To apply 4.2 to a proof of (4), suppose that $X$ is some topological space and that for some set $S$ and $p \in \operatorname{Fn}(S, 2)$,

$$
p \Vdash X \text { is perfect. }
$$

Fix $H \subseteq X$ a closed set. For each $x \in X$ let $\mathcal{V}_{x}$ be a neighborhood base at $x$. Since it is forced by $p$ that $H$ is a $G_{\delta}$, we may fix names $\left\{\tau_{n}: n<\omega\right\}$ such that

(i) for each $n<\omega, p \Vdash \tau_{n} \supseteq H$ is open,

(ii) $p \Vdash \bigcap_{n<\omega} \tau_{n}=H$.

For each $n<\omega$ fix an $n$-dowment $\mathcal{L}_{n}$ below $p$. For each $x \in H$ and $n<\omega$ we can find a maximal antichain $A_{x}^{n}$ below $p$ such that for each $g \in A_{x}^{n}$ there is a $V(g, x) \in \mathcal{V}_{g}$ such that $g \Vdash V(g, x) \in \tau_{n}$. For each $x \in H$ and $n<\omega$ fix $L_{n}(x) \in \mathcal{L}_{n}$ such that $L_{n}(x) \subseteq A_{x}^{n}$ and let $V_{n}(x)=\bigcap_{g \in L_{n}(x)} V(g, x)$. Finally, we let $V_{n}=\bigcup_{x \in H} V_{n}(x)$ and claim that $\bigcap_{n<\omega} V_{n}=H$. Clearly this will finish the proof.

So suppose not and fix $y \in X \backslash H$ such that $y \in \bigcap_{n<\omega} V_{n}$. For each $n<\omega$, pick $x_{n} \in H$ such that

(iii) $y \in V_{n}\left(x_{n}\right)$.

Since $p \Vdash y \notin \bigcap_{n<\omega} \tau_{n}$ there is an $f<p$ and an $N<\omega$ such that

(iv) $f \Vdash$ for each $i \geq N, y \notin \tau_{i}$.

By extending $f$ if necessary we may assume that if $n=|\operatorname{dom}(f)|$ then $n>N$. Now pick $g \in L_{n}\left(x_{n}\right)$ such that $g$ is compatible with $f$. Then since $g \in A_{x_{n}}^{n}, g \cup f \Vdash V_{n}\left(x_{n}\right) \subseteq \tau_{n}$, which contradicts (iii) and (iv).

\section{References}

[B] D. K. Burke, PMEA and first countable countably metacompact spaces, Proc. Amer. Math. Soc. 92 (1984), 455-460.

[C] J. Chaber, Metacompactness and the class of MOBI, Fund. Math. 91 (1976), 211-217.

[D] P. Davies, Nonperfect space with point-countable bases, Proc. Amer. Math. Soc. 77 (1979), 276-278. 
[vD] E. K. van Douwen, The integers and topology, in: K. Kunen and J. E. Vaughan (eds.), Handbook of Set-Theoretic Topology, North-Holland, Amsterdam, 1984, $111-167$.

[DTW] A. Dow, F. D. Tall and W. A. R. Weiss, New proofs of the consistency of the normal Moore space conjecture I, Topology Appl. 37 (1990), 33-51.

[F] W. G. Fleissner, Normal Moore spaces in the constructible universe, Proc. Amer. Math. Soc. 46 (1974), 294-298.

[FR] W. G. Fleissner and M. Reed, Paralindelöf spaces and spaces with a $\sigma$-locally countable base, Topology Proc. 2 (1977), 89-110.

[K] K. Kunen, Set Theory, An Introduction to Independence Proofs, North-Holland, Amsterdam, 1980.

[N1] P. Nyikos, A provisional solution to the normal Moore space problem, Proc. Amer. Math. Soc. 78 (1980), 429-435.

[N2] -, Countably metacompact, locally countable spaces in the constructible universe, Topology Appl., to appear.

[S] P. J. Szeptycki, Uncovering separation properties in the Easton model, preprint.

[T1] F. D. Tall, Set-theoretic consistence results and topological theorems concerning the normal Moore space conjecture and related problems, Dissertationes Math. 148 (1977)

[T2] -, Covering and separation properties in the Easton model, Topology Appl. 28 (1988), 155-163.

[W] S. Watson, Separation in countably paracompact spaces, Trans. Amer. Math. Soc. 290 (1985), 831-842.

Current address:

DEPARTMENT OF MATHEMATICS

UNIVERSITY OF TORONTO

TORONTO, ONTARIO

CANADA M5S 2M3
DEPARTMENT OF MATHEMATICS OHIO UNIVERSITY ATHENS, OHIO 45701-2979 U.S.A.

Received 11 September 1992;

in revised form 25 March and 5 May 1993 